\title{
A Compound Fuzzy Disturbance Observer Based on Sliding Modes and Its Application on Flight Simulator
}

\author{
Yunjie Wu, ${ }^{1}$ Youmin Liu, ${ }^{1}$ and Dapeng Tian ${ }^{2}$ \\ ${ }^{1}$ School of Automation Science and Electrical Engineering, Beihang University, Beijing 100191, China \\ ${ }^{2}$ Changchun Institute of Optics, Fine Mechanics and Physics, Chinese Academy of Science, Jilin 130033, China \\ Correspondence should be addressed to Youmin Liu; snakelym1985@163.com
}

Received 2 December 2012; Accepted 28 January 2013

Academic Editor: Peng Shi

Copyright (c) 2013 Yunjie Wu et al. This is an open access article distributed under the Creative Commons Attribution License, which permits unrestricted use, distribution, and reproduction in any medium, provided the original work is properly cited.

\begin{abstract}
A compound fuzzy disturbance observer based on sliding modes is developed, and its application on flight simulator is presented. Fuzzy disturbance observer (FDO) is an effective method in nonlinear control. However, traditional FDO is confined to monitor dynamic disturbance, and the frequency bandwidth of the system is restricted. Sliding mode control (SMC) compensates the highfrequency component of disturbance while it is limited by the chattering phenomenon. The proposed method uses the sliding mode technique to deal with the uncompensated dynamic equivalent disturbance. The switching gain of sliding mode control designed according to the error of disturbance estimation is a small value. Therefore, the proposal also helps to decrease the chattering. The validity of the proposal method is confirmed by experiments on flight simulator.
\end{abstract}

\section{Introduction}

Flight simulator simulates the attitude of aircraft and helps the ground experiments. High precision motion control is the key of a flight simulator, which influences the accuracy of simulation experiments. As a typical kind of servomotor system, the robustness against external nonlinear disturbances, time-varied characters, and modeling uncertainties is urgently required [1]. The disturbance observer (DOB) approach has been widely used as an effective robust method to compensate the disturbance and parameter variations from both environment and system [2,3]. The disturbance observer, which makes the system dynamics same as the nominal model in ideal condition, can absolutely compensate the equivalent disturbance regarding the external disturbance torque, modeling error, and other uncertain factors. However, this method is a linear one and equivalent to high-gain control, which may cause resonance in controlling elastic electromechanical systems [4]. Moreover, there exists huge modeling mismatch if the nonlinear characters of the system are ignored and the performance of the system is limited as the two elements are contradictive in stable controller designing.
Fuzzy method, as a nonlinear method, has been studied with the basic idea that a fuzzy logic system can well approximate arbitrary highly nonlinear system $[5,6]$. In previous work, fuzzy approach has been used in complex nonlinear areas such as facial pattern recognition [7], project management [8], and economic dispatch [9] and obtained good effect. Facing to the difficulties caused by nonlinear factors in control area, an apparent view is to use fuzzy tools to monitor and compensate these uncertainties. In some investigations, fuzzy method has been used in controller design [10, 11], filter design [12, 13], and observer design $[14,15]$. Thereinto, a fuzzy-disturbance-observer- (FDO-) based control suggested by Kim [14] inherits the advantages of disturbance observer and avoids the unstable factors when there exists huge difference between the nominal model and the actual system. Compared with the indirect and direct adaptive fuzzy approaches suggested by Chen et al. [15] and Ordonez and Passino [16], the FDO-based method guarantees the uniformly ultimately bounded of the disturbance observation error within a sufficiently small region by appropriate choice of the design parameter without the requirement of a supervisory or a robustifying controller. FDO deals with nonlinear problems in motion control 
systems and is optimal to industrial application. However, FDO still has difficulty in compensating high-frequency component of a nonlinear disturbance sufficiently.

Sliding mode control (SMC) can inhibit high-frequency disturbance by switching control value, which also causes chattering phenomenon. SMC is an effective approach to deal with nonlinear systems $[17,18]$; therefore, an apparent solution is to combine SMC and disturbance observer together. SMC is used as an outer loop controller in some investigations while disturbance observer deals with the compensation of disturbance $[19,20]$. In these conditions, the SMC needs to improve the tracking performance as well as inhibiting the disturbance simultaneous. As a result, the chattering phenomenon is hardly decreased as the switching gain keeps affecting. Meanwhile, the upper bound on unknown disturbances is generally considered as constant estimation in order to meet the conditions for the existence of generalized sliding mode [21, 22]. Therefore, if the upper bound is underestimated, the tracking performance of motor servo system is difficult to improve and the system is possibly unstable. Moreover, the switching gain of SMC is designed conservatively and the chattering alleviation is also limited. In previous work, sliding mode disturbance observer has also been proposed as an independent branch which employs the sliding mode technique to estimate the lumped disturbance rather than to do the position control directly [23, 24], and some intelligent methods have also been employed to observe the varying disturbances and estimate the upper bound accordingly $[25,26]$. However, the conservative is still a great problem as the switching gain should be larger than the unknown upper bound, and most of intelligent units are not sufficiently sensitive to the chattering in the output of the controller.

In this paper, a compound fuzzy disturbance observer (CFDO) based on sliding modes is proposed, and the task of disturbance compensation is divided into two parts. Lowfrequency disturbance is compensated by FDO while highfrequency disturbance is treated by SMC. As low-frequency component is the main part of equivalent disturbance, SMC deals with only the secondary part of the disturbance. Consequently, the switching gain of SMC may be designed as a relative small value, and the chattering alleviation is achieved. The proposed method comprehended the advantages of both FDO and SMC. By using this method, the equivalent disturbance can be compensated more accurately, and the resonance caused by traditional methods in controlling elastic electromechanical systems can be avoided. Compared with the traditional proposed DOB and FDO schemes, CFDO has better robustness when there exists large nonlinear factors, decreases the modeling mismatch, and extends the frequency bandwidth of the systems. The model of disturbance is not required when the CFDO is designed. As a typical kind of servo motor system, the theoretic results in flight simulator can be used in other servo motion control systems.

The brief outline of the paper is as follows. In Section 2, the fuzzy disturbance observer is introduced. In Section 3, the control problem is formulated. Then, the structure and design of CFDO are proposed. In Section 4, experimental results are

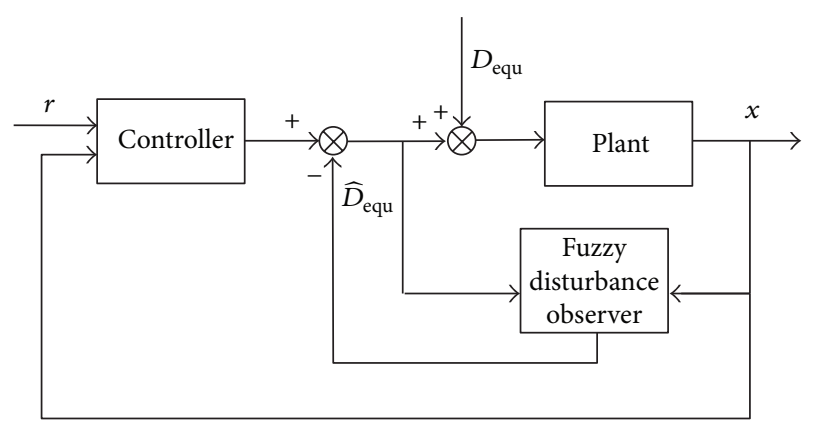

FIGURE 1: Structure of the control system with fuzzy disturbance observer.

included to support the theoretical work. Finally, the paper is concluded in Section 5.

\section{Fuzzy Disturbance Observer}

Consider a system described as

$$
\begin{gathered}
\dot{x}_{1}=x_{2}, \\
\dot{x}_{2}=\left[\alpha\left(x_{2}\right)+\Delta A(x)\right]+\left[\beta\left(x_{2}\right)+\Delta B(x)\right] u+d_{\text {ext }},
\end{gathered}
$$

where $u$ denotes controller output, $x=\left(x_{1}, x_{2}\right)^{T}$ denotes state vector of the system, $\alpha\left(x_{2}\right)$ and $\beta\left(x_{2}\right)$ are decided by nominal model, $\Delta A(x)$ and $\Delta B(x)$ denote the internal unknown time-varying nonlinear dynamics, and $d_{\text {ext }}$ denotes generalized external disturbance. In the viewpoint of disturbance observer, both internal uncertainty and external disturbance can be defined as equivalent disturbance, and the system can be written as

$$
\dot{x}_{2}=\alpha\left(x_{2}\right)+\beta\left(x_{2}\right)\left(u+D_{\text {equ }}\right),
$$

where $D_{\text {equ }}=\left[\Delta A(x)+\Delta B(x) u+d_{\text {ext }}\right] / \beta\left(x_{2}\right)$ is the nonlinear equivalent disturbance in controller output terminal and cannot be described by mathematical expressions in general. A fuzzy logic is used as a solution to approximate the nonlinear disturbance as an FDO.

Figure 1 shows the structure of FDO. From the structure, the equivalent disturbance can be compensated sufficiently, and the system has the same dynamics as the nominal model in the case of the fuzzy system $\widehat{D}_{\text {equ }}$ follows the disturbance $D_{\text {equ }}$ completely.

In order to achieve the compensation, the estimated value can be obtained by fuzzy logic system, which is described briefly here. The fuzzy inference engine uses the fuzzy IFTHEN rules to perform a mapping from an input compact set $x=\left(x_{1}, x_{2}, \ldots, x_{n}\right)^{T} \in R^{n}$ to an output variable $z \in R$. The fuzzy rules can be described as

$$
R^{i} \text { : If } x_{1} \text { is } K_{1}^{i} \text { and } \cdots \text { and } x_{n} \text { is } K_{n}^{i} \text {, then } z \text { is } z_{i} \text {, }
$$

where $K_{1}^{i}, K_{2}^{i}, \ldots, K_{n}^{i}$ are fuzzy variables and $z_{i}$ is a singleton number. By using product inference engine, center-average 
defuzzifier, and singleton fuzzifier, the output of the fuzzy system can be expressed as

$$
z(x)=\frac{\sum_{i=1}^{M} z_{i}\left(\prod_{j=1}^{n} \mu_{K_{j}^{i}}\left(x_{j}\right)\right)}{\sum_{i=1}^{M}\left(\prod_{j=1}^{n} \mu_{K_{j}^{i}}\left(x_{j}\right)\right)}=\hat{\theta}^{T} \xi(x),
$$

where $\mu_{K_{j}^{i}}\left(x_{j}\right)$ is membership function value of the fuzzy variable $x_{j}, M$ is the number of fuzzy rules, $\hat{\theta}^{T}=\left(z_{1}, z_{2}, \ldots\right.$, $\left.z_{M}\right)$ denote adjustable parameter vector, and $\xi(x)=\left(\xi_{1}(x)\right.$, $\left.\xi_{2}(x), \ldots, \xi_{M}(x)\right)^{T}$ denote fuzzy basis functions defined by

$$
\xi^{i}=\frac{\prod_{j=1}^{n} \mu_{K_{j}^{i}}\left(x_{j}\right)}{\sum_{i=1}^{M}\left(\prod_{j=1}^{n} \mu_{K_{j}^{i}}\left(x_{j}\right)\right)} .
$$

In order to design an FDO, a tuning method must be developed for the adjustable parameter vector. Consider the following dynamic system (7):

$$
\dot{\mu}=-\sigma \mu+\sigma x_{2}+\alpha\left(x_{2}\right)+\beta\left(x_{2}\right)\left(u+\widehat{D}_{\text {equ }}\right) .
$$

Define disturbance observation error as $\varsigma \equiv x_{2}-\mu$. Obviously, $\varsigma \rightarrow 0\left(\mu \rightarrow x_{2}\right)$ implies that the estimated disturbance value $\widehat{D}_{\text {equ }}$ approaches the actual but unknown disturbance $D_{\text {equ. }}$.

The disturbance observation error $\varsigma$ is uniformly ultimately bounded within a region, which size can be kept arbitrarily small, if the adjustable parameter vector of the FDO is tuned by (8) and bounded. To ensure the FDO outputs zero signal in case of $D_{\text {equ }}=0$ (perfect matching), the adjustable parameter vector is set to zero as (9). The correlated proof can be found in [14]. Consider

$$
\begin{gathered}
\dot{\hat{\theta}}=\gamma \varsigma \xi(x, u), \\
\widehat{\theta}(0)=0,
\end{gathered}
$$

As a typical servo system, a flight simulator system can be described as

$$
J_{n} \ddot{\theta}+B_{n} \dot{\theta}=u+d,
$$

where $d$ is the nonlinear equivalent disturbance in controller output terminal, $J_{n}$ is the nominal inertia, and $B_{n}$ is the nominal damping. Define $x_{1}=\theta, x_{2}=\dot{\theta}$, it is significant that the system (10) is a special case of system (2), and the FDO can be designed as is stated previously.

The FDO makes system response the same as the nominal model. Compared with DOB, FDO-based control has advantages on the robust stability and static performance when the system lacks mechanical stiffness. Unfortunately, some flight simulator systems are elastic electromechanical systems, and the use of DOB is restricted. Therefore, the FDO helps to improve the static performance and robustness of these flight simulators.

However, the nonlinear disturbance, such as friction, is composed of the components of many frequencies. The highfrequency components limit the system performance and the effect of FDO is limited in practice [27]. Therefore, disturbance compensation method with better effect is expected.

\section{Compound Fuzzy Disturbance Observer}

Consider the flight simulator system (10) with the help of FDO, it can be described as (11), where $\widehat{d}$ is the estimated disturbance by FDO. The difference between the observer value $d$ and the equivalent disturbance $\widehat{d}$ should be compensated. Define a secondary system as (12), where $u_{c}$ is the objective control value to make $e \rightarrow 0$. In this case, $u_{c}$ is expected to $d-\widehat{d}$. Consider

$$
\begin{gathered}
J_{n} \ddot{\theta}+B_{n} \dot{\theta}=u+d-\widehat{d}, \\
J_{n} \ddot{\theta}_{n}+B_{n} \dot{\theta}_{n}=u+u_{c}, \\
e=\theta-\theta_{n} .
\end{gathered}
$$

Define the sliding surface as (14), where $c=B_{n} / J_{n}$. Then the objective turns into making $w \rightarrow 0$. The control value is designed as (15), where $\kappa$ is a positive constant gain, $\psi$ is the positive switching gain, $\varepsilon$ is a small constant that communicates with the boundary layer near the sliding mode surface, and $\operatorname{sat}(\bullet)$ is the saturation function defined as (16):

$$
\begin{gathered}
w=\dot{e}+c e, \\
u_{c}=\kappa w+\psi \text { sat }\left(\frac{\psi w}{4 \varepsilon}\right), \\
\operatorname{sat}(\bullet)= \begin{cases}1, & \bullet \geq 1 \\
\bullet, & |\bullet|<1 \\
-1, & \bullet \leq-1 .\end{cases}
\end{gathered}
$$

According to (11) (15), there is (17). Define a positive definite Lyapunov candidate (18); its time derivative is obtained as (19):

$$
\begin{gathered}
J_{n} \dot{w}=J_{n} \ddot{\theta}_{n}+B_{n} \dot{\theta}_{n}-\left(J_{n} \ddot{\theta}+B_{n} \dot{\theta}\right) \\
=-\kappa w-\psi \operatorname{sat}\left(\frac{\psi w}{4 \varepsilon}\right)+\hat{d}-d, \\
V(w)=\frac{1}{2} J_{n} w^{2}, \\
\dot{V}(w)=w J_{n} \dot{w} \leq-\kappa w^{2}-w \psi \operatorname{sat}\left(\frac{\psi w}{4 \varepsilon}\right)+|w||\widehat{d}-d|, \\
\psi \geq|\widehat{d}-d| .
\end{gathered}
$$

Suppose that (20) is satisfied; the analysis of (19) is discussed as follows.

If $|w| \geq 4 \varepsilon / \psi$ and $\psi \geq|\widehat{d}-d|$, there is (21). From (21) and considering $|w| \geq 4 \varepsilon / \psi$, there is (22):

$$
\begin{gathered}
\dot{V}(w)=-\kappa w^{2}-|w|(\psi-|\widehat{d}-d|) \\
\leq-\kappa w^{2}=-2 \frac{\kappa}{J_{n}} V(w) \leq 0, \\
\lim _{t \rightarrow \infty}|w(t)| \leq \frac{4 \varepsilon}{|\widehat{d}-d|} .
\end{gathered}
$$


If $|w| \leq 4 \varepsilon / \psi$ and $\psi \geq|\widehat{d}-d|$, there is (23). According to the solution of the differential inequality, there is (24) and (25):

$$
\begin{aligned}
& \dot{V}(w)=-\kappa w^{2}-\frac{\psi^{2}|w|^{2}}{4 \varepsilon}+|w||\widehat{d}-d| \\
& \leq-\kappa w^{2}-\frac{\psi^{2}|w|^{2}}{4 \varepsilon}+|w| \psi \\
&=-\kappa w^{2}-\frac{1}{\varepsilon}\left(\frac{\psi|w|}{2}-\varepsilon\right)^{2}+\varepsilon \\
& \leq-\kappa w^{2}+\varepsilon=-2 \frac{\kappa}{J_{n}} V(w)+\varepsilon \\
& w^{2}(t) \leq w^{2}(0) \exp \left(-\frac{2 \kappa}{J_{n}} t\right)+\frac{\varepsilon}{\kappa}\left[1-\exp \left(-\frac{2 \kappa}{J_{n}} t\right)\right], \\
& \lim _{t \rightarrow \infty}|w(t)| \leq \sqrt{\frac{\varepsilon}{\kappa}} .
\end{aligned}
$$

Consequently, if (20) is satisfied, $w$ exponentially converges and satisfied (25). By the definition of $w$, e exponentially converges and satisfied (26). Therefore, the compound disturbance observer can be designed if (20) is satisfied:

$$
\lim _{t \rightarrow \infty}|e(t)| \leq \frac{1}{c} \sqrt{\frac{\varepsilon}{\kappa}} .
$$

The expression (20) should be satisfied; that is, the switching gain is related to the difference between equivalent disturbance and observer value of FDO. From the process of FDO design, the difference aforementioned are decided by the disturbance observation error $\varsigma$. Therefore, the estimation of upper bound which is used to design sliding mode controller can be got by $\varsigma$. When the disturbance observation error disappears after some time, the switching gain of the SMC can be kept in a small value. And further, the chattering phenomenon can be decreased. The expression (27) gives the description of the switching gain in this paper, where $\eta$ is the magnification coefficient that can be tried in practice. The whole structure of CFDO is showed in Figure 2. Consider

$$
\psi=\eta \varsigma
$$

\section{Experiment Results}

In order to test the effect of the proposed method, an experiment is implemented by using a three-axis flight simulator shown in Figure 3. The optical-electrical encoder with resolution of 0.0007 degrees is employed as the position sensor. The program of control algorithm is written with $\mathrm{C}$ language based on Windows-RTX real-time system in an industrial computer (Advantech IPC 610), which connects with the servo drivers by a 16-bit D/A convertor of PCI bus. The control cycle is $0.001 \mathrm{~s}$.

As in the previous discussion, FDO monitors both the internal and the external disturbance so that each axis of the

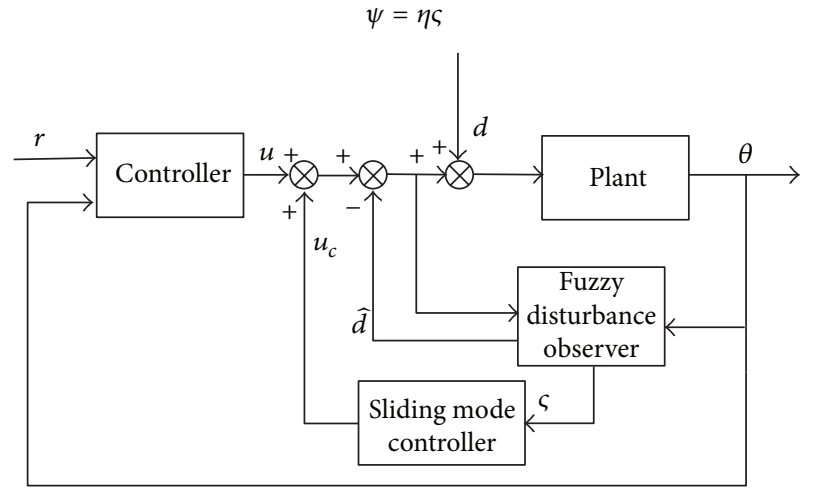

FIGURE 2: Structure of the control system with compound fuzzy disturbance observer.

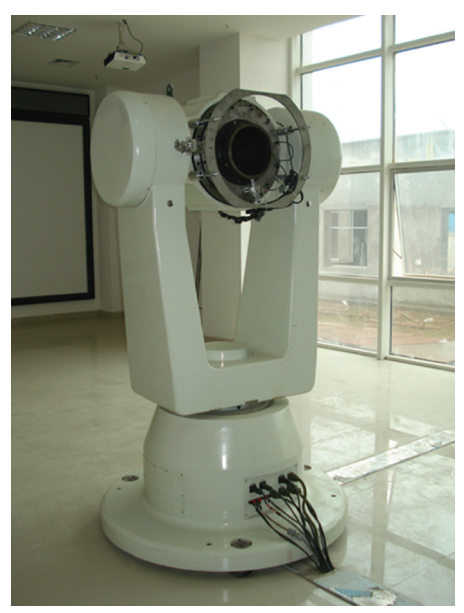

FIGURE 3: The three-axis flight simulator.

flight simulator can be designed independently. Therefore, the pitch axis is chosen herein to verify the method. The controller design is based on the parameters which are acquired by identifying the flight simulator. The parameters of the nominal model are identified as $J_{n}=0.0172, B_{n}=$ 0.0948. The fitting curves for frequency characteristics of actual plant and nominal model are shown in Figure 4. A PD controller is used as a position controller and $K_{p}=0.65$, $K_{d}=0.03$. The membership functions shown in Figure 5 are selected for the premise parts of the FDO. Other factors employed in the experiment are given as follows: $\sigma=15$, $\gamma=350, \kappa=10$, and $\eta=1.1$. A nonlinear tracking differential estimator is used to get differential value [28], and the nonlinear tracking differential gain is $g_{v}=400$. The accuracy of the static position, the stationarity of the static velocity, and the dynamic frequency are the most important indexes to a flight simulator. Generally, the static position accuracy can be guaranteed by a stable disturbance observer.

Figure 6 shows the curves of the steady-state speed under $\mathrm{DOB}, \mathrm{FDO}$, and $\mathrm{CFDO}$ schemes, respectively. In this case, the reference signal is constant and the response curves tracking with the velocity of $1^{\circ} / \mathrm{s}$ as well. All of the systems can reach the position command value ultimately. 


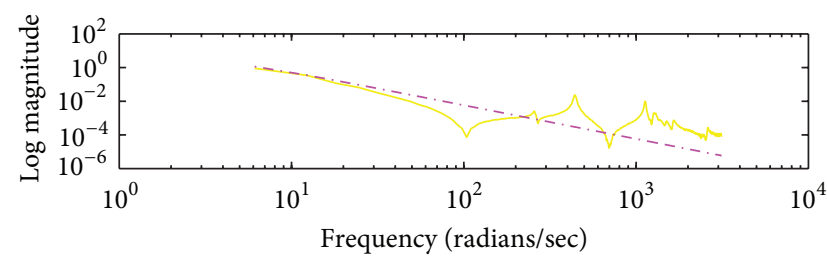

(a)

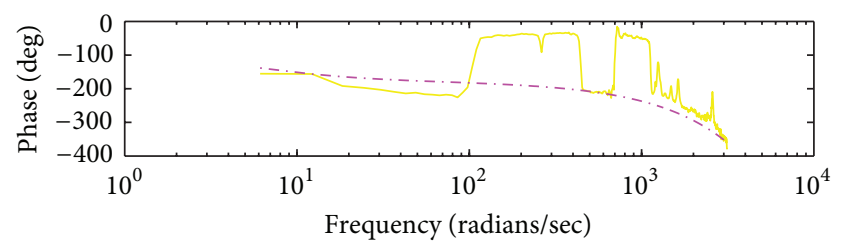

(b)

FIGURE 4: The fitting curves for frequency characteristics of actual plant and nominal model.

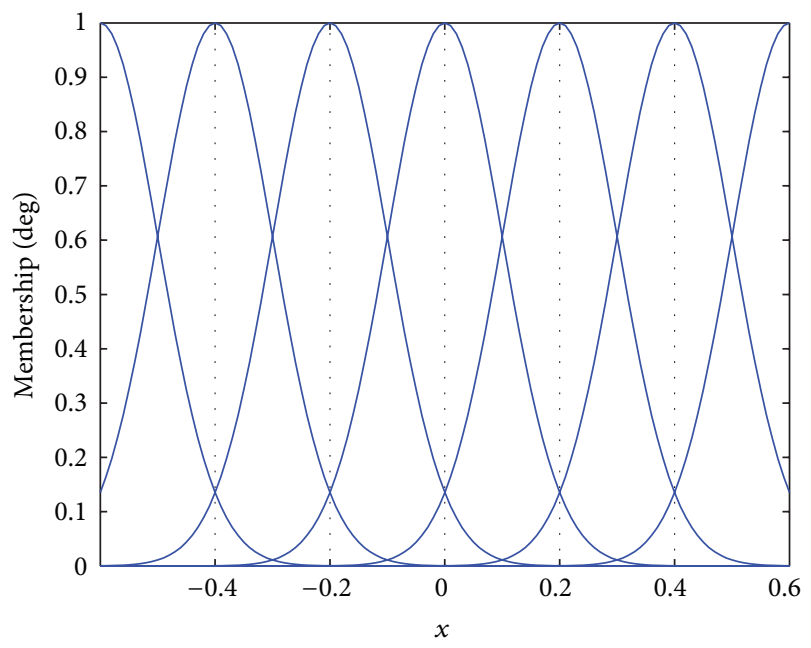

FIGURE 5: Gaussian membership functions.

However, the DOB scheme has a oscillatory speed because of the influence of elasticity. The FDO and CFDO schemes can keep a stable speed while tracking and it is the effect of FDO as the sliding mode part almost has no effect in static condition. Compared with traditional DOB scheme, the proposed method has better robustness as FDO is a nonlinear approach and decreases the modeling mismatch. Evidently, reducing the effect of DOB helps to decrease the oscillation while restricting the performance of the system.

Figure 7 compares the tracking error and the control value of the FDO and CFDO schemes when the reference signal is described as $2 \sin (2 \pi \cdot 0.5 t)$. The figure shows that the maximum tracking error under the FDO scheme approximately equals $0.037 \mathrm{deg}$ while that decreases to $0.028 \mathrm{deg}$ using the proposed CFDO. The tracking error indicates that the system with CFDO has better dynamic performance than the system with only FDO. Furthermore, the control value of the CFDO system does not exhibit obvious chattering phenomenon compared with the value of the FDO system. The system performance is effected by FDO primarily, and the sliding mode controller whose switching gain is restricted in a small range only assists secondarily.

Figure 8 shows the tracking error and the control value of the two systems when the reference signal is described as $2 \sin (2 \pi \cdot 4 t)$. High-frequency disturbance, treated by $\mathrm{SMC}$, is the main problem in this environment. The figure

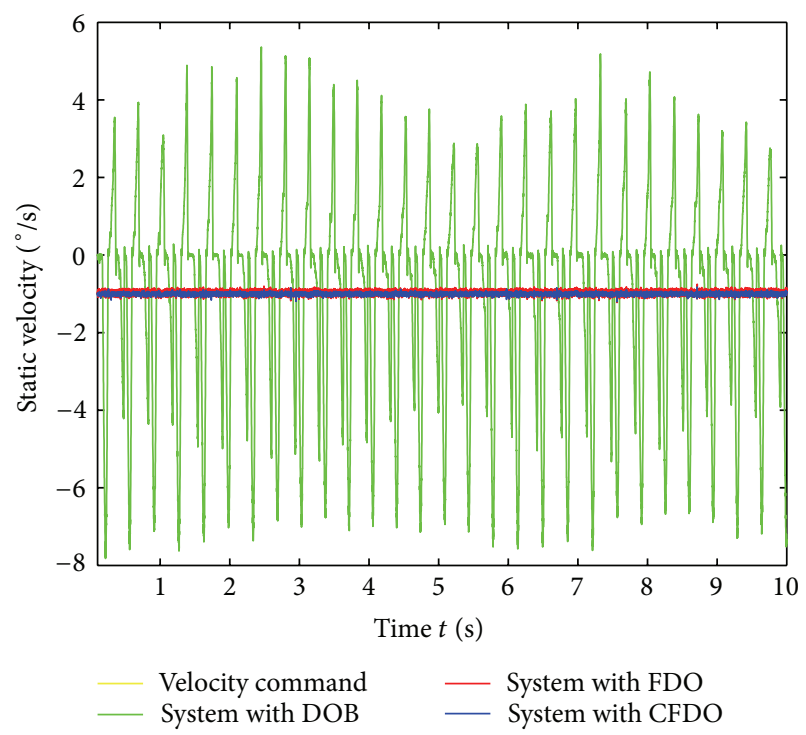

Figure 6: The curves of the steady-state speed under DOB, FDO, and CFDO schemes.

shows that the maximum tracking error under the FDO scheme approximately equals $0.652 \mathrm{deg}$ while that decreases to 0.203 deg using the proposed CFDO. From the comparison of the two error curves, the CFDO system has better dynamic performance and the frequency range is extended with the help of SMC. Inevitably, chattering phenomenon can hardly be alleviated for the SMC need to recover rapidly.

Neither the steady-state error of FDO nor the error of CFDO is influenced by the extra constant disturbance, as FDO compensates the constant disturbance over time. However, under the condition of small time-varying disturbance like Figure 8, CFDO has better performance than FDO as the sliding mode controller deals with the high-frequency disturbance rapidly. According to Figures 7 and 8, changes of working frequency have less influence under CFDO scheme than under FDO scheme; that is, the system with CFDO has better robustness than the system with FDO.

The part of sliding mode controller compensates the highfrequency component of disturbance and improves not only the robustness but also the dynamic performance of the system, meanwhile, the FDO deals with the low-frequency 


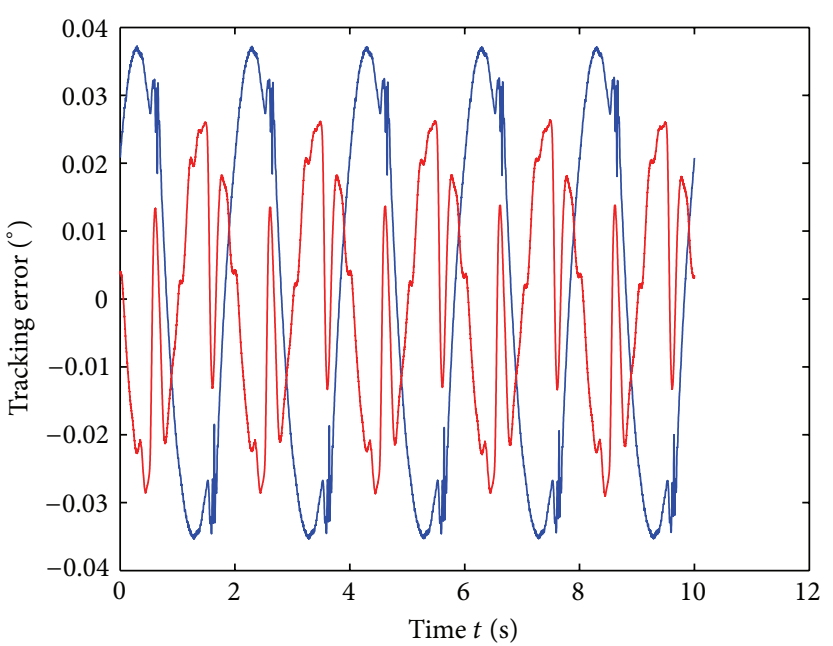

— System with FDO

— System with CFDO

(a)

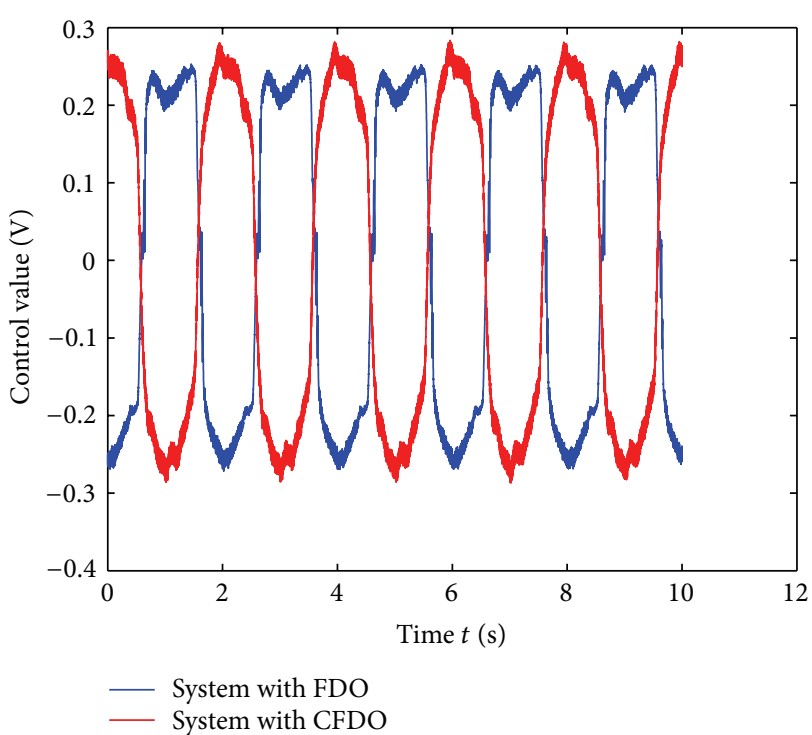

(b)

FIGURE 7: The comparison of error curves and control value in the case of sin input $(A=2, f=0.5)$.

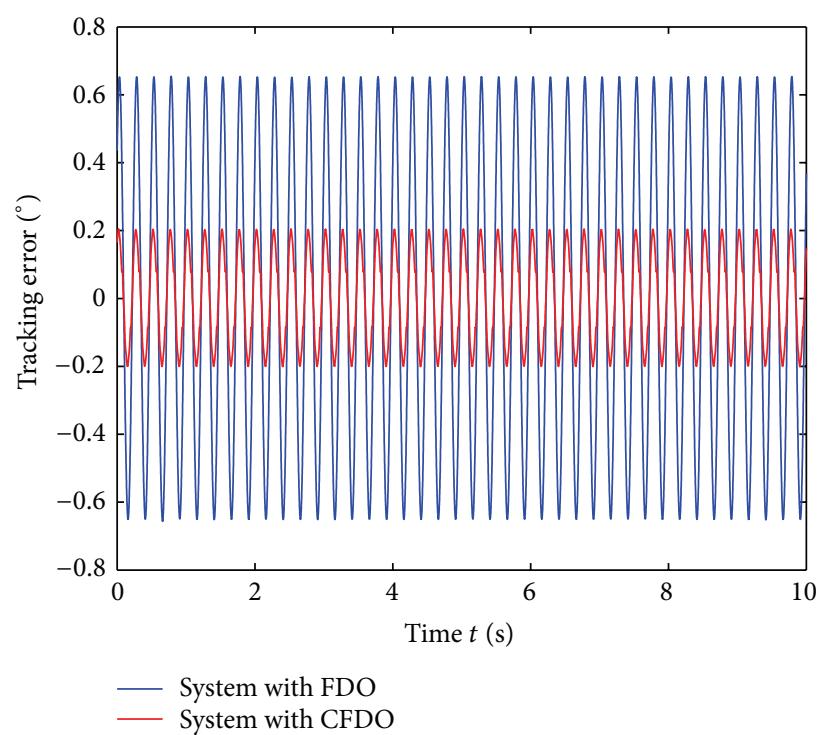

(a)

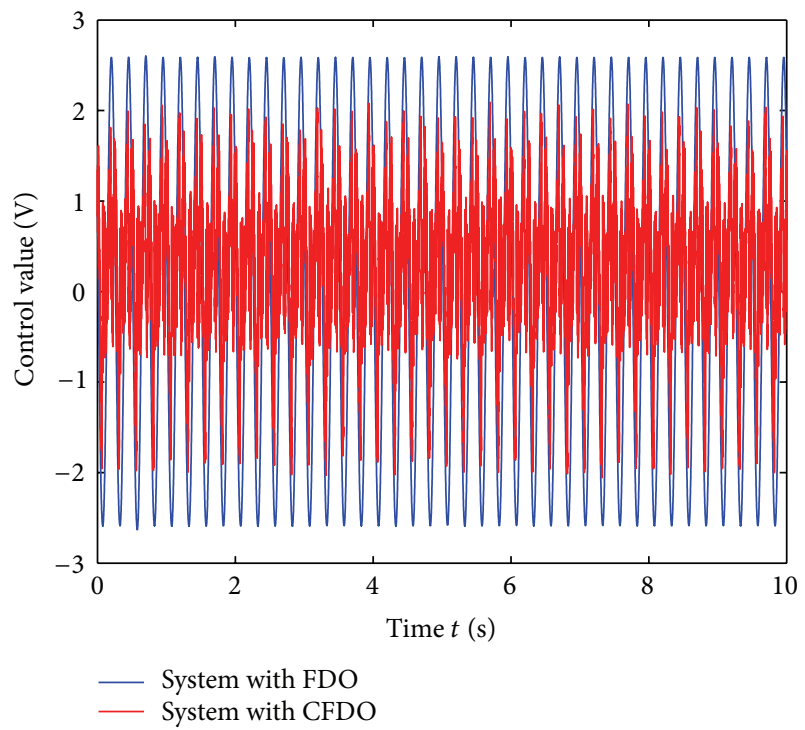

(b)

FIGURE 8: The comparison of error curves and control value in the case of sin input $(A=2, f=4)$.

component of disturbance and helps to weaken the chattering. In consequence, they help each other to compensate the equivalent disturbance.

To a flight simulator system, the references are often slow-varying signals, which can be seen as static signals, in most part of an aircraft trajectory. In the last part of the trajectory, there will be fast-varying signals. CFDO meets the demand of these systems perfectly: FDO guarantees the accuracy, chattering is limited in most part of time, and SMC helps the system to respond fast while facing enormous variations.

\section{Conclusion}

This paper proposes a compound fuzzy disturbance observer based on sliding modes. The equivalent disturbance is sufficiently compensated by using the proposed CFDO when there exists huge modeling mismatch, and the disadvantages of FDO and SMC are avoided. The performance, especially the accuracy, of the system is improved. The switching gain of sliding mode controller is designed by disturbance estimation error, and the reduction of switching gain helps to weaken the chattering. 
The method has been validated by experiments. By using the proposed method, the maximum tracking error decreases from $0.037 \mathrm{deg}$ to $0.028 \mathrm{deg}$ in low-frequency condition with the reference of $2 \sin (2 \pi \cdot 0.5 t)$, and from 0.652 deg to $0.203 \mathrm{deg}$ in high-frequency condition with the reference of $2 \sin (2 \pi \cdot 4 t)$. Most servo motion control systems have similar characteristics as flight simulator; therefore, the theoretic results are able to be extended to other relational fields such as mechanical arm systems, camera tracking systems, and other servo motion control systems, especially those with highprecision requirement and deficient mechanical stiffness.

However, the coefficient about the fuzzy disturbance observer error can only be decided by trying in practice and an unsuitable parameter may lead to performance degradation or make the system unstable. In future work, better ways to decide switching gain will be studied and the fuzzy adjustable method will be optimized.

\section{Acknowledgment}

This work was supported by the National Natural Science Foundation of China (Grant no. 91216304).

\section{References}

[1] Q. Liu and L. Er, "Disturbance observer based robust tracking control of high precision flight simulator," Journal of Beijing University of Aeronautics and Astronautics, vol. 29, no. 2, pp. 181184, 2003 (Chinese).

[2] T. R. Grochmal and A. F. Lynch, "Precision tracking of a rotating shaft with magnetic bearings by nonlinear decoupled disturbance observers," IEEE Transactions on Control Systems Technology, vol. 15, no. 6, pp. 1112-1121, 2007.

[3] K.-S. Kim, K.-H. Rew, and S. Kim, "Disturbance observer for estimating higher order disturbances in time series expansion," IEEE Transactions on Automatic Control, vol. 55, no. 8, pp. 19051911, 2010.

[4] T. Umeno, T. Kaneko, and Y. Hori, "Robust servosystem design with two degrees of freedom and its application of novel motion control of robot manipulators," IEEE Transactions on Industrial Electronics, vol. 40, no. 5, pp. 473-485, 1993.

[5] X. Zeng and M. G. Singh, "Approximation theory of fuzzy systems-MIMO case," IEEE Transactions on Fuzzy Systems, vol. 3, no. 2, pp. 219-235, 1995.

[6] L. Wu, X. Su, P. Shi, and J. Qiu, "Model approximation for discrete-time state-delay systems in the TS fuzzy framework," IEEE Transactions on Fuzzy Systems, vol. 19, no. 2, pp. 366-378, 2011.

[7] S. Y. Cho, C. W. Ting, and C. Quek, "Thermal facial pattern recognition for personal verification using fuzzy cmac model," International Journal of Innovative Computing, Information and Control, vol. 7, no. 1, pp. 203-222, 2011.

[8] M. J. Wang and M. F. Yang, "Application of signed distances method to integrate linear programming for fuzzy multiobjective project management," International Journal of Innovative Computing, Information and Control, vol. 7, no. 1, pp. 237252, 2011.

[9] T. Niknam, H. D. Mojarrad, and M. Nayeripour, "A new hybrid fuzzy adaptive particle swarm optimization for nonconvex economic dispatch," International Journal of Innovative
Computing, Information and Control, vol. 7, no. 1, pp. 189-202, 2011.

[10] X. Su, P. Shi, L. Wu, and Y.-D. Song, "A novel control design on discrete-time Takagi-Sugeno fuzzy systems with time-varying delays," IEEE Transactions on Fuzzy Systems, 2012.

[11] L. Wu, X. Su, P. Shi, and J. Qiu, "A new approach to stability analysis and stabilization of discrete-time T-S fuzzy timevarying delay systems," IEEE Transactions on Systems, Man, and Cybernetics B, vol. 41, no. 1, pp. 273-286, 2011.

[12] M. H. Bahari, A. Karsaz, and N. Pariz, "High maneuvering target tracking using a novel hybrid Kalman filter-fuzzy logic architecture," International Journal of Innovative Computing, Information and Control, vol. 7, no. 2, pp. 501-510, 2011.

[13] X. Su, P. Shi, L. Wu, and Y.-D. Song, "A novel approach to filter design for T-S fuzzy discrete-time systems with time-varying delay," IEEE Transactions on Fuzzy Systems, vol. 20, no. 6, pp. 1114-1129, 2012.

[14] E. Kim, "A fuzzy disturbance observer and its application to control," IEEE Transactions on Fuzzy Systems, vol. 10, no. 1, pp. 77-84, 2002.

[15] B. S. Chen, H. J. Uang, and C. S. Tseng, "Robust tracking enhancement of robot systems including motor dynamics: a fuzzy-based dynamic game approach," IEEE Transactions on Fuzzy Systems, vol. 6, no. 4, pp. 538-552, 1998.

[16] R. Ordonez and K. M. Passino, "Stable multi-input multi-output adaptive fuzzy/neural control," IEEE Transactions on Fuzzy Systems, vol. 7, no. 3, pp. 345-353, 1999.

[17] T. C. Lin, S. W. Chang, and C. H. Hsu, "Robust adaptive fuzzy sliding mode control for a class of uncertain discretetime nonlinear systems," International Journal of Innovative Computing, Information and Control, vol. 8, no. 1, pp. 347-359, 2012.

[18] Q. Khan, A. I. Bhatti, M. Iqbal, and Q. Ahmed, "Dynamic integral sliding mode control for SISO uncertain nonlinear systems," International Journal of Innovative Computing, Information and Control, vol. 8, no. 7, pp. 4621-4633, 2012.

[19] Y. Wu, W. Le, and D. Tian, "Application of sliding mode control based on disturbance observer on high performance flight motion simulator," in Proceedings of the IEEE International Conference on Automation and Logistics (ICAL '07), pp. 26952699, August 2007.

[20] A. Hace, K. Jezernik, and A. Sabanovic, "SMC with disturbance observer for a linear belt drive," IEEE Transactions on Industrial Electronics, vol. 54, no. 6, pp. 3402-3412, 2007.

[21] Z. L. He, J. F. Wu, G. H. Sun, and C. Gao, "State estimation and sliding mode control of uncertain switched hybrid systems," International Journal of Innovative Computing, Information and Control, vol. 8, no. 10, pp. 7143-7156, 2012.

[22] K. D. Young, V. I. Utkin, and U. Ozguner, "A control engineer's guide to sliding mode control," IEEE Transactions on Control Systems Technology, vol. 7, no. 3, pp. 328-342, 1999.

[23] X. Chen, S. Komada, and T. Fukuda, "Design of a nonlinear disturbance observer," IEEE Transactions on Industrial Electronics, vol. 47, no. 2, pp. 429-437, 2000.

[24] S. J. Lopez, O. C. Nieto, and J. I. C. Oria, "Non-parametric modeling of uncertain hyperbolic partial differential equations using pseudo-high order sliding mode observers," International Journal of Innovative Computing, Information and Control, vol. 8, no. 3, pp. 1501-1521, 2012.

[25] M. Zhihong, X. H. Yu, K. Eshraghian, and M. Palaniswami, "A robust adaptive sliding mode tracking control using an $\mathrm{RBF}$ 
neural network for robotic manipulators," in Proceedings of the IEEE International Conference on Neural Networks, pp. 24032408, December 1995.

[26] V. Panwar, "Asymptotic trajectory tracking for a robot manipulator using RBF neural network and adaptive bound on disturbances," in Proceedings of the International Conference on Mechanical and Electrical Technology (ICMET '10), pp. 156-160, September 2010.

[27] Z. Jamaludin, H. van Brussel, and J. Swevers, "Friction compensation of an XY feed table using friction-model-based feedforward and an inverse-model-based disturbance observer," IEEE Transactions on Industrial Electronics, vol. 56, no. 10, pp. 38483853, 2009.

[28] D. Tian, D. Yashiro, and K. Ohnishi, "New nonlinear tracking differential-estimators: theory and practice," International Journal of Innovative Computing, Information and Control, vol. 8, no. 12, pp. 8257-8271, 2012. 


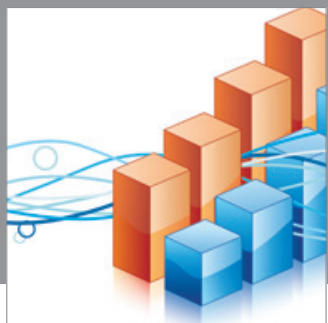

Advances in

Operations Research

mansans

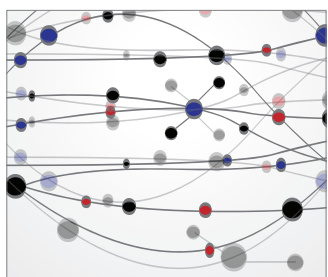

The Scientific World Journal
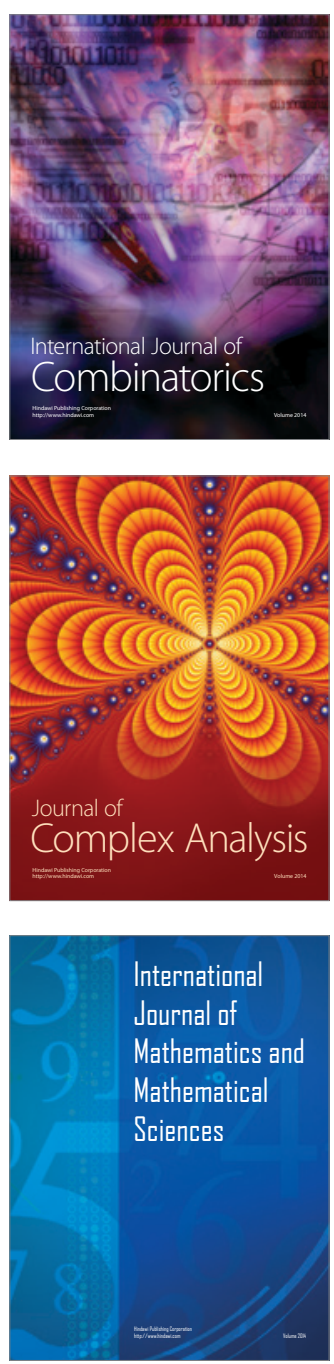
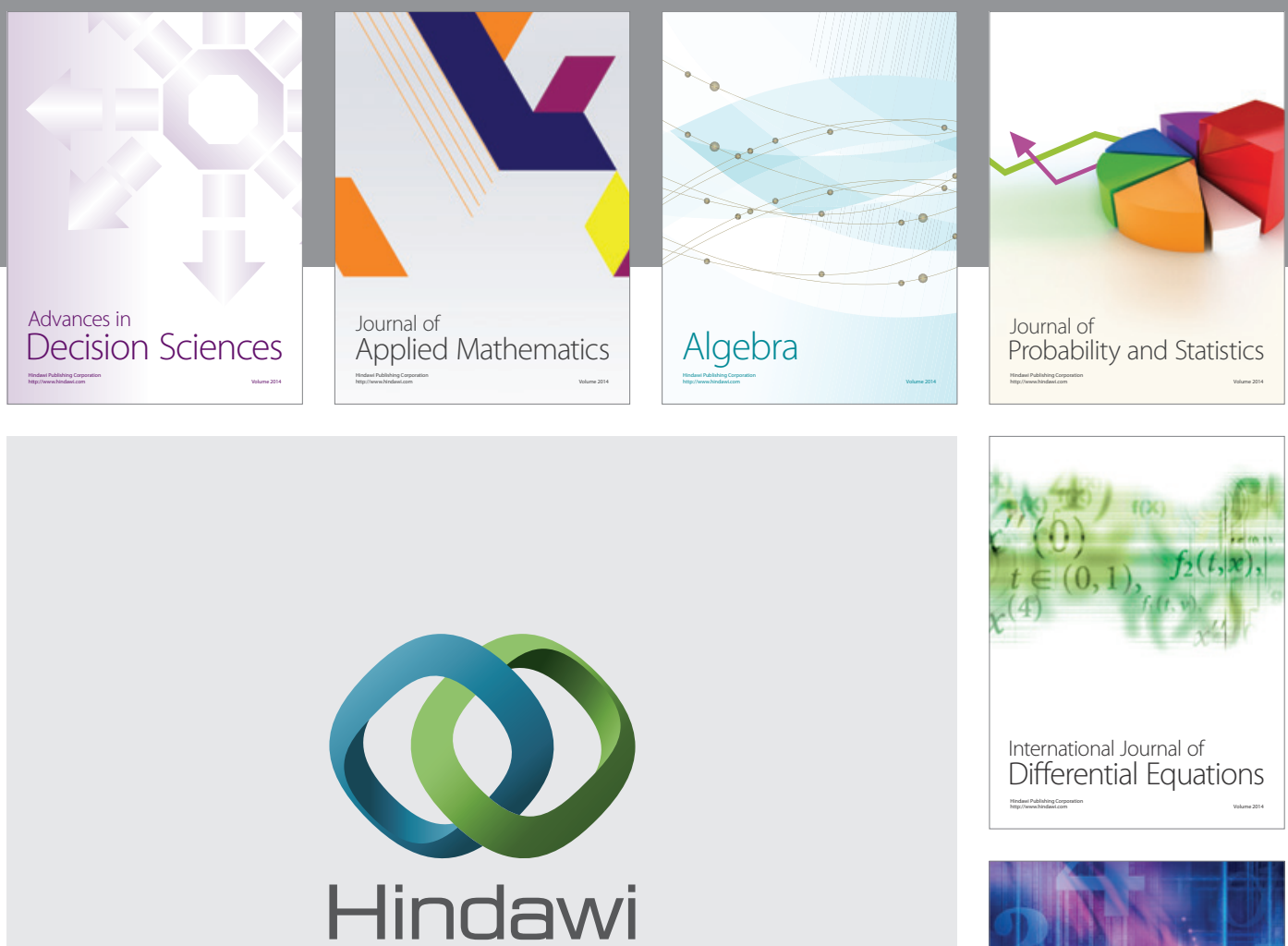

Submit your manuscripts at http://www.hindawi.com
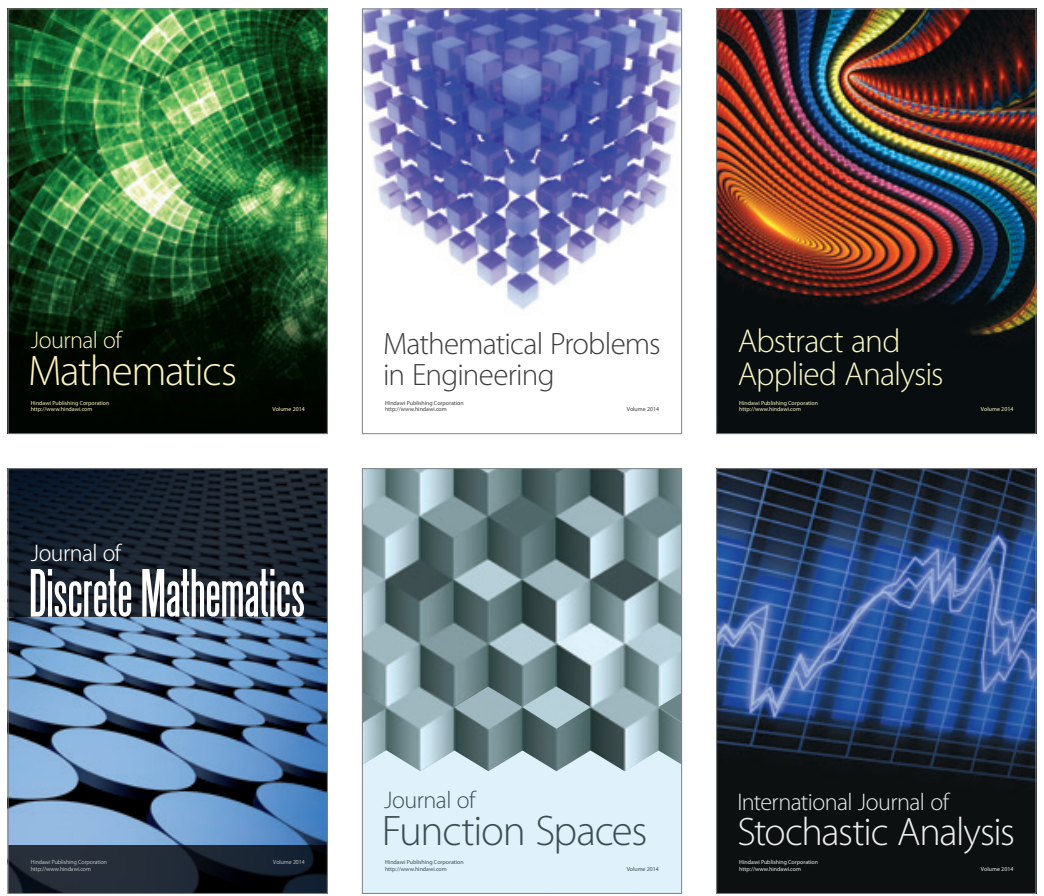

Journal of

Function Spaces

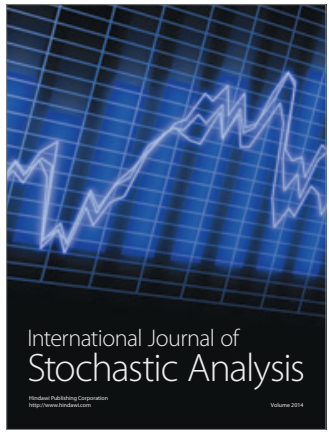

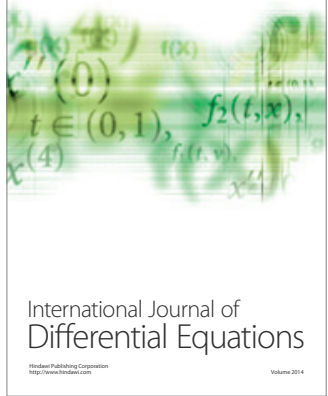
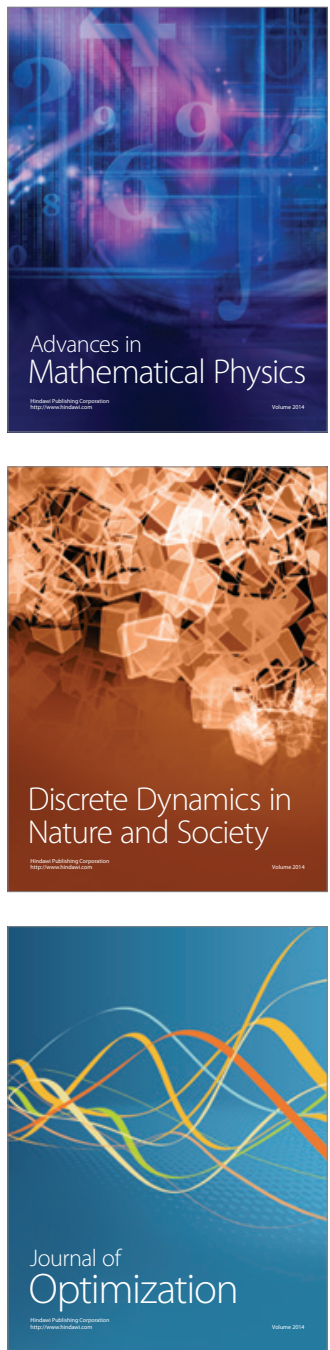